\title{
The Location Routing Problem using Electric Vehicles with Constrained Distance
}

\author{
Abdullah Almouhanna - Carlos L. \\ Quintero-Araujo • Javier Panadero • \\ Angel A. Juan • Banafsheh Khosravi . \\ Djamila Ouelhadj
}

Received: date / Accepted: date

\begin{abstract}
The introduction of Electric Vehicles (EVs) in modern fleets facilitates a shift towards greener road transportation practices. However, the driving ranges of EVs are limited by the duration of their batteries, which raises some operational challenges. This paper discusses the Location Routing Problem with a Constrained Distance (LRPCD), which is a natural extension of the Location Routing Problem when EVs are utilized. A fast multistart heuristic and a metaheuristic are proposed to solve the LRPCD. The former combines biased-randomization techniques with the well-known Tillman's heuristic for the Multi-Depot Vehicle Routing Problem. The latter incorporates the biased-randomized approach into the Variable Neighborhood Search (VNS) framework. A series of computational experiments show that the multi-start heuristic is able to generate good-quality solutions in just a few seconds, while the biased-rendomized VNS metaheuristic provides higherquality solutions by employing more computational time.
\end{abstract}

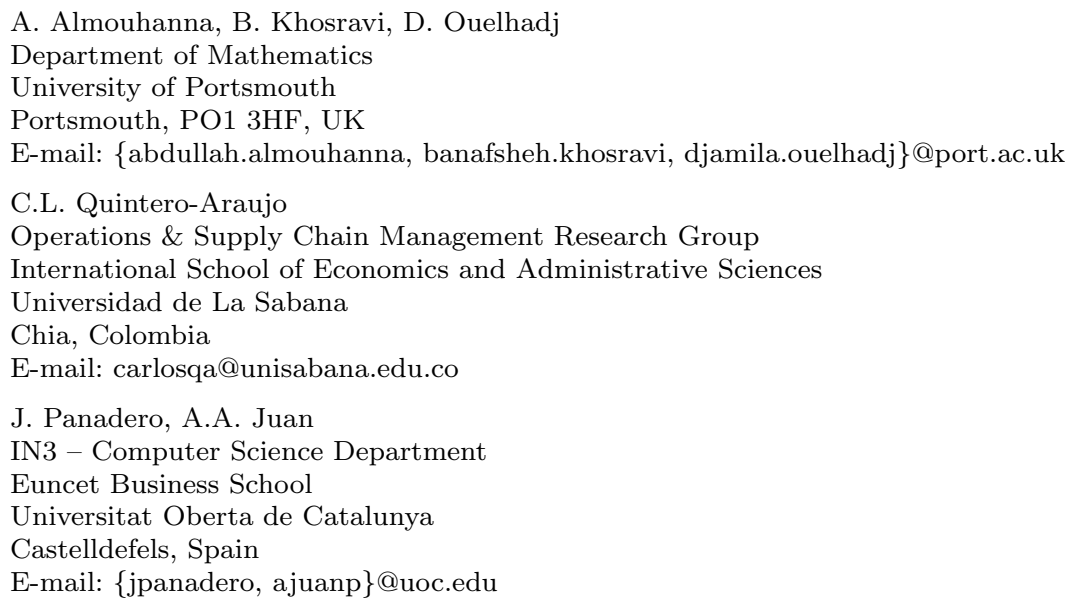


Keywords Location Routing Problem - Green Logistics • Variable Neighborhood Search · Biased Randomization

\section{Introduction}

Transportation is one of the main activities in supply chain management. It has a huge impact on the customers' satisfaction (Crainic, 2000), and it also plays an important role in the generation of $\mathrm{CO}_{2}$ and greenhouse-gas (GHG) emissions and related externalities, including: air pollution, noise, and traffic congestions (Juan et al, 2016). Road transportation alone is responsible for about $18 \%$ of total GHG emissions in the EU (Hill et al, 2011). Higher percentages of $\mathrm{CO}_{2}$ emissions have been reported in other parts of the world, such as the United States of America (United States Environmental Protection Agency, 2014). Therefore, it becomes necessary to consider more ecological power sources for fueling vehicles, as well as horizontal cooperation strategies to make road transportation activities more sustainable (Pérez-Bernabeu et al 2015, Quintero-Araujo et al, 2017). Electric Vehicles (EVs) and plug-in hybrid electric vehicles (PHEV) are examples of growing green technologies and some governments are making noticeable efforts to promote the use of these types of vehicles (Mattila and Antikainen, 2011). Therefore, there has been more recent attempts by researchers and industries to consider EVs in transport.

Regarding more traditional internal-combustion-engine vehicles (ICEVs) and PHEVs, it is assumed that they have unlimited driving-range capabilities since they can refuel at any service station along their route. On the contrary, driving ranges for EVs are limited by the amount of electricity stored in their batteries, since they cannot quickly recharge en-route. In fact, the EV issues related to long charging times and short driving ranges are recognized as a major challenge (Juan et al, 2014). Despite being the fastest charging option, rapid chargers are not a realistic option for delivery trucks since: (i) fast charging stations account for only $14 \%$ of total charging stations in Europe, (ii) there are four different standards for rapid chargers, which implies that not all charging stations are suitable for all types of electric trucks, (iii) while charging larger batteries overnight may be feasible even with a Level 2 charger - especially for the delivery van - fleets with less time to charge may require more power all at once. This level of power can put a much larger strain on the electrical grid than passenger vehicle chargers, and can result in time-intensive negotiations with local utilities and costly demand charges (GreenBiz, 2019). Thus, as stated by Ferreira et al (2011), EVs are the next big step in the automobile industry, but continue to have a limited autonomy associated with the long charging times, limited charging stations, and undeveloped smart grid infrastructures. Similar arguments can be found in Achtnicht et al (2012), Wirasingha et al (2008), and Chan et al (2009). Also, it was reported by the Institute for Social-Ecological Research (2017) that the reduced range will remain the main issue concerning electric mobility. Moreover, the maximum range is impacted by different factors such as weather conditions (Yuksel and 
Michalek, 2015), loads and speed (Wager et al, 2016). According to Chellaiah and $\mathrm{R}(2017)$, the maximum driving range in for the EVs is between $120 \mathrm{~km}$ and $390 \mathrm{~km}$. These experts claim that "this (situation) is not likely to change considerably in the medium term". With EVs becoming more prevalent, an efficient routing of fleets with driving-range limitations (i.e., time or distance constraints) is emerging as a new issue in the transportation industry. Hence, it is still more realistic and viable to consider limited driving range for EVs due to inadequate supporting infrastructure for EVs.

In recent literature, some studies consider locating intra-route facilities to swap or recharge EVs batteries simultaneously with determining route plans. However, the single-depot assumption of these studies is too restrictive to represent real-world problems. In this paper, we address this gap and consider a unique combination of planning aspects. This study not only considers opening multiple depots and allocating customers to them, but also determines simultaneously locating depots and routing EVS, which have limited driving range. It is also considerable that our model is completely operational as a single-echelon problem whereas the recent studies with intra-route facilities for swapping and recharging ignore replenishment of the facilities and they should be extended to consider another echelon for facility replenishment to be complete.

This paper addresses the LRP with a Constrained Distance (LRPCD), which is uses EVs in the location and routing decisions. Figure 1 illustrates different stages of solving the LRPCD. Firstly, depots are selected. Then, customers are assigned to depots. Finally, vehicle routes are computed. The last sub-figure (d) shows how the routing plan might be significantly altered due to the introduction of driving range constraints.

The contribution of this research study is threefold. Firstly, the research work proposes a new LRPCD optimisation model with a unique combination of planning aspects compared to existing literature on LRP using EVs. Secondly, two novel solution methods are developed, including a fast multi-start biased-randomized heuristic and a biased-randomized Variable Neighborhood Search (VNS) metaheuristic. The proposed heuristic is a fast and efficient algorithm which iteratively determines location decisions and routing decisions based on an embedding classic heuristic (Tillman, 1969), instead of the prevalent well-known savings heuristic (Clarke and Wright, 1964), combined with biased randomization technique. The biased-randomized VNS meta-heuristic is based on the VNS Search framework (Mladenović and Hansen, 1997) which incorporates biased randomization technique. Thirdly, the effectiveness of the developed solution methods is demonstrated by comparing their performance using existing benchmark instances for the LRP and newly generated instances for the LRPCD. The results have showed that the heuristic approach is able to generate reasonably good solutions in a matter of seconds, which can be very useful in real-time situations. The biased-randomized VNS meta-heuristic provides better solutions by employing employing more computational time.

The remainder of this paper is organized as follows. A literature review on the LRPCD is provided in Section 2, The problem definition and an op- 

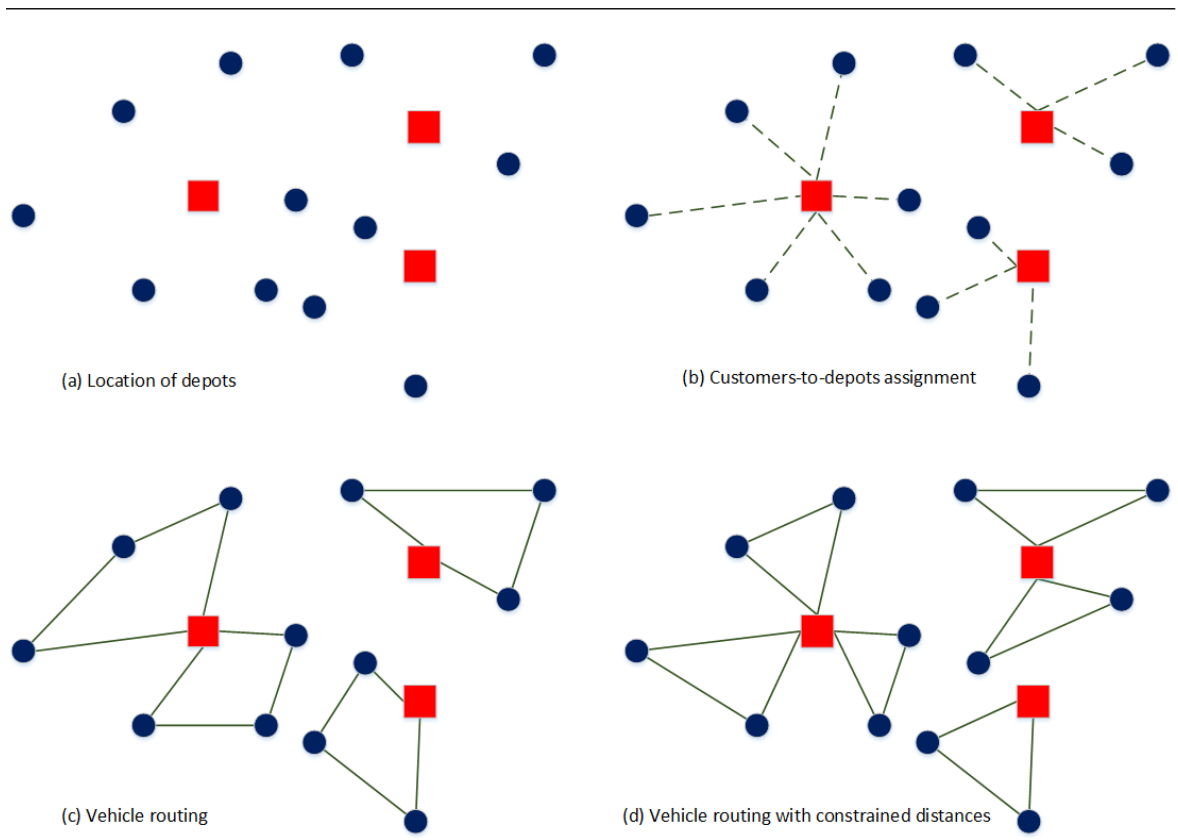

Fig. 1 An illustrative example of Green LRP.

timisation model of the LRPCD is presented in section 3 Section 4 introduces outhe fast multi-start biased-randomized heuristic, while the biasedrandomized VNS meta-heuristic is described in section 5. The experimental design for the computational analysis is described in Section 6. Next, the computational results and their analysis are discussed. Finally, conclusions and suggestions for future work are given in Section 7

\section{Literature Review}

The LRP has a wide range of applications including telecommunication systems, food and drinks distribution, waste collection, and disaster logistics (Nagy and Salhi, 2007). The benefits derived from taking into account routing decisions while locating facilities has been quantified for the first time by Salhi and Rand (1989). They have shown that solving a location problem and a routing problem separately does usually lead to sub-optimal solutions. Despite the importance of the LRP in supply chain management, the number of published studies available in the literature is scarce compared to other logistics problems e.g., vehicle routing problems and arc routing problems.

In recent years, there has been an increasing interest to study routing and location problems with EVs in order to reduce GHG emission. We refer the reader to Bektaş et al (2016) for an overview of significant studies on Green VRP (GVRP), and Koç et al (2016) for recent studies on Heterogeneous VRP 
(HVRP). In this section, we provide an overview of related research on LRPs with a constrained distance in presence and absence of EVs.

Therefore, we investigate two streams of research related to our study on LRP using EVs with a constrained distance. The first stream investigates the classic LRP to integrate location and routing decisions simultaneously with route-length constraints in different practical scenarios, where no EV is used. The other stream, which is relatively new, is related to locating intra-route facilities for battery swapping, or recharging stations for EVS, or refueling Alternative Fuel Vehicles (AFVs) that have emerged due to rise of EV technology and its role in reducing greenhouse gas emissions and providing less dependence on traditional fuels. The mentioned studies consider an LRP problem with distance constraints as vehicles have limited battery driving range.

Regarding the LRPCD in the first stream of studies, the route-length constraint is considered when customers have to be served before or within a certain time. Jacobsen and Madsen (1980), and Singh and Shah (2004) consider, respectively, a location-routing model for the distribution of newspapers in Denmark, and collection of tendupatta leaves in India. Jacobsen and Madsen (1980) consider route-length constraints because newspapers must be delivered to the last customer before 11 a.m., whereas Singh and Shah (2004) use them because some items have to be delivered within a few hours from picking. Jacobsen and Madsen (1980) develop a heuristic which solves the problem by switching between location and routing, while Singh and Shah (2004) solve the proposed model using a CPLEX model.

The working hours or days are another main reason to apply the routelength constraints. Lin et al (2002) address the delivery of telecommunication bills in Hong Kong. The mentioned constraints are applied based on the working hours. Lin et al (2002) propose a two-stage heuristic; the initial solution and the improvement routing. The initial solution is generated by a heuristic, whereas routing is solved using simulated annealing. Jouzdani and Fathian (2014) model a dairy supply chain in Iran as an LRP with route-length constraints regarding the working hours. The model is solved by using the LINGO optimizer.

Caballero et al (2007) address the waste collection in Spain and apply the LRP model with route-length constraints to represent the working days of a driver. The problem is solved using a metaheuristic algorithm based on the tabu search. Chan and Baker (2005) address the LRP with tour-length limits, asymmetrical distances, and both pick-up and delivery. A maximum tour length is applied to represent realistic crew working hours per day. A heuristic procedure is described to solve the LRP in two steps. First, the minimum spanning forests is used to locate the regional depots and assign demand nodes to depots. Second, the classical savings heuristic Clarke and Wright, 1964) is applied to route the fleet of homogeneous vehicles.

Another main reasons for applying the route-length constraints are security and safety. Regarding security, Murty and Djang (1999) and Sarıçiçek and Akkus (2015) each apply an LRP model including a route-length limitation, respectively, to the training of National Guard units at the U.S. Army, and 
to the hub-location of border security in Turkey. Murty and Djang (1999) use a heuristic hierarchical decomposition strategy to break the overall problem into several manageable subproblems, so that it can be solved in successive stages. Sarıçiçek and AkkuS (2015) use CPLEX to solve the problem. With regard to safety, Rath and Gutjahr|(2014) and Coutinho-Rodrigues et al (2012) apply the distance constraints during natural disasters to minimize the total distance of delivering relief goods and to minimize the total travel distance to shelters, respectively. Rath and Gutjahr (2014) propose a multi-objective LRP to provide relief goods to affected people. The model is solved using a VNS metaheuristic. Coutinho-Rodrigues et al (2012) develop a multi-objective LRP model to design an evacuation plan in Portugal. The solution is determined by solving the associated mixed integer linear programming model.

In e-business, the route-length limitation is used to increase the service quality and the profit. Aksen and Altinkemer (2008) introduce the constraint of a maximum driving distance on the LRP model to increase service quality when switching from traditional retailing to e-retailing. Lagrangian relaxation is proposed to generate lower bounds, whereas a heuristic method is used to find feasible solutions. In space exploration, Ahn et al (2012) address the LRP with a distance constraint to maximize the sum of profits that can be obtained by visiting sites under resource consumption constraints. Two solution methods are proposed including a branch-and-price and a three-phase heuristic method combined with a greedy randomized adaptive search procedure.

The second stream of studies on LRP with distance constraints consider locating intra-route facilities to replenish vehicles (EVs or AFVs) by swapping or recharging batteries, in addition to routing plans for EVs or AFVs. Yang and Sun (2015) and Hof et al (2017) study battery swap stations location-routing problem for EVs which aims to determine the locations of stations, as well as a routing plan. Yang and Sun (2015) propose two metaheuristics including a four-phase Adaptive Large Neighborhood Search (ALNS) algorithm, and a heuristic which combines tabu search with an iterated modified Clarke and Wright saving method. Hof et al (2017) has successfully extended a solution method for VRP with intermediate stops in their proposed Adaptive Variable Neighborhood Search (AVNS). Ylldı et al (2016) develop a branch and price approach to solve routing and refueling stations location problem for AFVs.

Schiffer and Walther (2017) and Schiffer and Walther (2018) investigate location-routing problem with recharging facilities for EVs. Schiffer and Walther (2017) study the electric LRP with time windows and partial recharging, where simultaneous siting decisions for charging stations, partial recharging, and recharging both at charging stations and at customer location are allowed. They investigate the impact of considering variants of the model with different combinations of objective functions including minimizing total distance, number of vehicles, number of charging stations, which are implemented using Gurobi. Schiffer and Walther (2018) present a robust LRP using EVs with time windows and partial recharging. They introduce uncertainty to customer patterns in terms of spatial customer distribution, demand, and service time windows. A parallelized hybrid approach consisting of ALNS and Dynamic 
Programming is developed to solve the problem. More recently, Schiffer et al (2018) have extended the LRP with intra-route facilities to consider combined facilities where different replenishment services including replenishing energy, or freight, or both are provided. They propose a hybrid ALNS and a local search which is enhanced by a lower bounding procedure to explore facility configurations efficiently at an early stage.

To compare the characteristics of recent LRP formulations using EVs with our model, we can highlight the following. Previous studies investigate singledepot location-routing problem that considers simultaneous decisions on routing EVs and locating intra-route facilities. In the research work presented in this paper, we consider locating multiple depots, allocation of customers to depots, and routing EVs simultaneously, which have limited driving range. There are two advantages to our problem compared to the mentioned studies. Firstly, it is more realistic and viable to model and solve the problem as an LRP using EVs with a constrained distance. As the issue of limited driving range still exists for EVs due to insufficient supporting infrastructure for EVs and lack of unified battery standards for various EVs, as identified by several researchers (Yang and Sun (2015); Yıldı et al (2016); Schiffer and Walther (2018). Secondly, our problem definition has this benefit that it can be operational fully on a single echelon, but the other LRP studies with intra-route facilities ignore replenishment of the intra-route facilities by solving the problem as a single-echelon problem.

\section{Problem Description and Optimization Model}

The LRPCD model is defined on a complete, weighted, and undirected graph $G=(V, E, C)$, where $V$ is a set of nodes (representing the subset $I$ of potential depot locations and the subset $J$ of customers) and $E$ is the set of undirected edges. Traversing each edge $(i, j) \in E$ has an associated cost $c_{i j}=c_{j i}>0$. Also, $K$ is a set of homogeneous vehicles, each of them with a loading capacity $q>0$ and a maximum driving range $d>0$. Each vehicle incurs a fixed cost $F$. Moreover, it is assumed that all vehicles are shared by all depots (i.e., no depot has a specific fleet) and each edge $e \in E$ satisfies the triangle inequality. Each customer's demand $D_{j}$ is deterministic and known in advance. The capacity of each depot $Q_{i}$ and its opening cost $O_{i}$ are also given. These depots might have equal or different capacities. Each customer must be served by a single vehicle departing from the depot to which the customer has been allocated. A solution for the LRP consists of determining which depots must be opened, defining the customer allocation to the available depots, and generating vehicle routes to serve the customers from their corresponding depot. The following constraints must be satisfied.

1. The total demand of customers assigned to one depot must not exceed its capacity.

2. Each route begins and ends at the same depot.

3. Each vehicle performs at most one trip. 
4. Each customer is only served by one vehicle (split deliveries are not allowed).

5. Total demand of customers visited by one vehicle fits its loading capacity.

6. The route length must not exceed the given distance constraint.

The proposed LRPCD optimisation model is an extension of the LRP model by Prins et al (2007). The notation is given as below.

Sets

$-V:$ Set of nodes, $V=I \cup J$.

$-I$ : Set of potential depot nodes.

$-J$ : Set of customers to be served.

$-E$ : Set of edges connecting nodes.

$-K$ : Set of vehicles (fleet size is considered to be virtually unlimited).

Parameters

- $O_{i}$ : Fixed cost of opening a depot at node $i$.

- $Q_{i}$ : Capacity of depot at node $i$.

- $D_{j}$ : Demand of customer at node $j$.

$-q$ : Loading capacity of each vehicle.

- F : Fixed cost per vehicle used.

$-c_{i j}$ : Traveling cost for edge $(i, j)$.

$-\bar{d}$ : Maximum distance allowed for each vehicle.

Decision variables

$-x_{i j k}$ : is equal to 1 if vehicle $k$ is used to cover the edge from customer $i$ to customer $j$, and 0 otherwise.

$-y_{i}$ : is equal to 1 if a depot is located at node $i$ and 0 otherwise.

- $U_{i}$ : is an arbitrary real number.

$-z_{i j}$ : is equal to 1 if customer $j$ is served by depot $i$, and 0 otherwise.

Then, the LRPCD can be formulated as follows:

$$
\min \quad \sum_{i \in I} O_{i} y_{i}+\sum_{i \in V} \sum_{j \in V} \sum_{k \in K} c_{i j} x_{i j k}+\sum_{i \in I} \sum_{j \in J} \sum_{k \in K} F x_{i j k}
$$


subject to

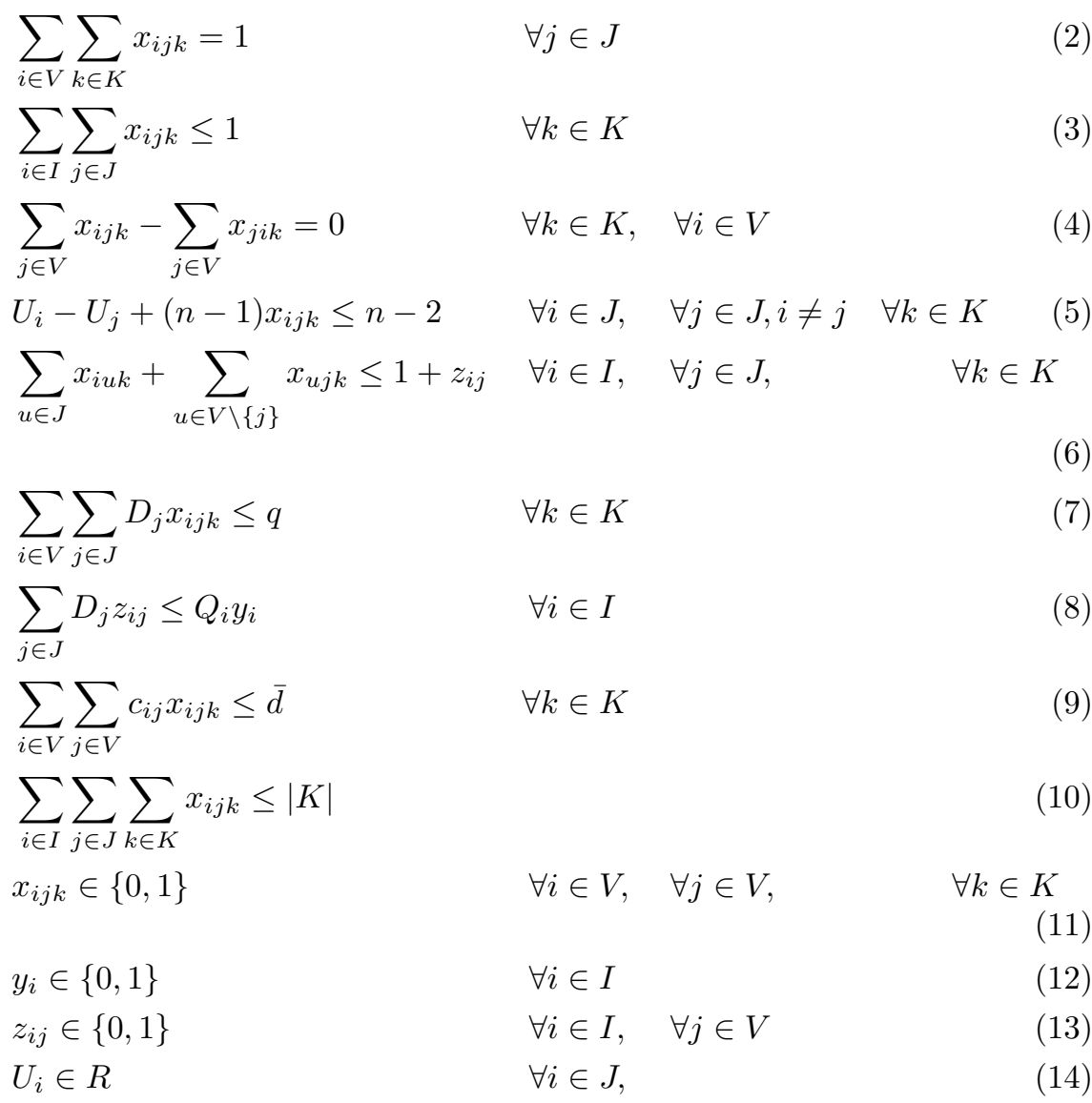

The objective function (1) aims to minimize the total cost, which includes the opening cost of depots, the variable distance-based cost of vehicles, and the fixed cost of using vehicles. Constraints (2) are the routing constraints that guarantee each customer has to be visited exactly once by a single vehicle. Constraints (3) ensure that all routes have to start and end at a depot, whereas constraints (4) are the connectivity constrains to ensure that every vehicle leaves each customer after it has been served. The sub-tour elimination constraints are defined by constraints (5). Constraints (6) ensure that a customer can be allocated to a depot only if there is a route for that depot. Constraints (7) and (8) specify that the capacity of each vehicle and the capacity of each depot should never be exceeded, respectively. Constraints (9) guarantee that the route length of each vehicle does not exceed the maximum distance constraint. Constraints 10 limit the number of vehicles to be used. Constraints (11) to (14) define the decision variables. 


\section{A Fast Multi-Start Biased-Randomized Heuristic}

In this section, a Multi-Start Biased-Randomized Heuristic (MSBRH) is proposed. As discussed in detail by Grasas et al (2017), biased-randomization techniques select the next constructive movement from a list of candidate moves. The elements of this list have different probabilities of being selected according to some priority criterion (usually given by the constructive heuristic). The main idea behind biased randomization is the introduction of a nonuniform random process that enhances the behavior of a greedy constructive heuristic. Applications of biased-randomized algorithms to different optimization problems can be found, for instance, in Dominguez et al (2014), De Armas et al (2017), or De Armas et al (2018).

MSBRH algorithm is composed of two stages. In the first stage, some depots are selected to be opened among the list of potential candidates. Once the depots to be opened are selected, the LRPCD is reduced to a MDVRP. The extended savings heuristic (ECWH) proposed by Tillman $(1969)$ is applied to allocate customers to opened depots and find an initial routing solution. This stage is repeated for different combinations of selected depots. The best solutions found during the first stage are then improved throughout the second stage, which includes two levels. In the global level, a biased-randomized version of the extended savings heuristic is applied to the initial solution resulted from the first stage. Whereas, in the local level a biased-randomized version of the classical savings heuristic is employed to improve the routes associated with each depot, as proposed by Juan et al (2011a). An overview of the procedure is presented in Figure 2, and more low-level details are provided next.

\subsection{First Stage: Selection of Promising Solutions}

The first stage of the proposed approach consists of a fast generation of several feasible and promising solutions for the LRPCD. Each of these solutions is obtained by the following procedure. In the first step, we determine a lower and an upper bound $(L B$ and $U B$ ) for the number of depots to be opened. The lower bound is calculated as the quotient between the total demand and the highest depot capacity. The upper bound ranges between $30 \%$ and $40 \%$ of the potential depots, as suggested in Nagy and Salhi (1996). At this point, all combinations of $m$ depots are tested, with $L B \leq m \leq U B$. For each of these combinations, the LRPCD is reduced to a MDVRP and solved by the ECWH.

The ECWH begins with an initial solution in which each customer is assigned to the nearest depot. Then, the solution is improved by joining customers together on a merged route in order to minimize the total travel distance. The customer routes are then assigned to the depot associated with this improvement. The customers selected to be joined are those with the maximum savings, where the following conditions must be satisfied:. 


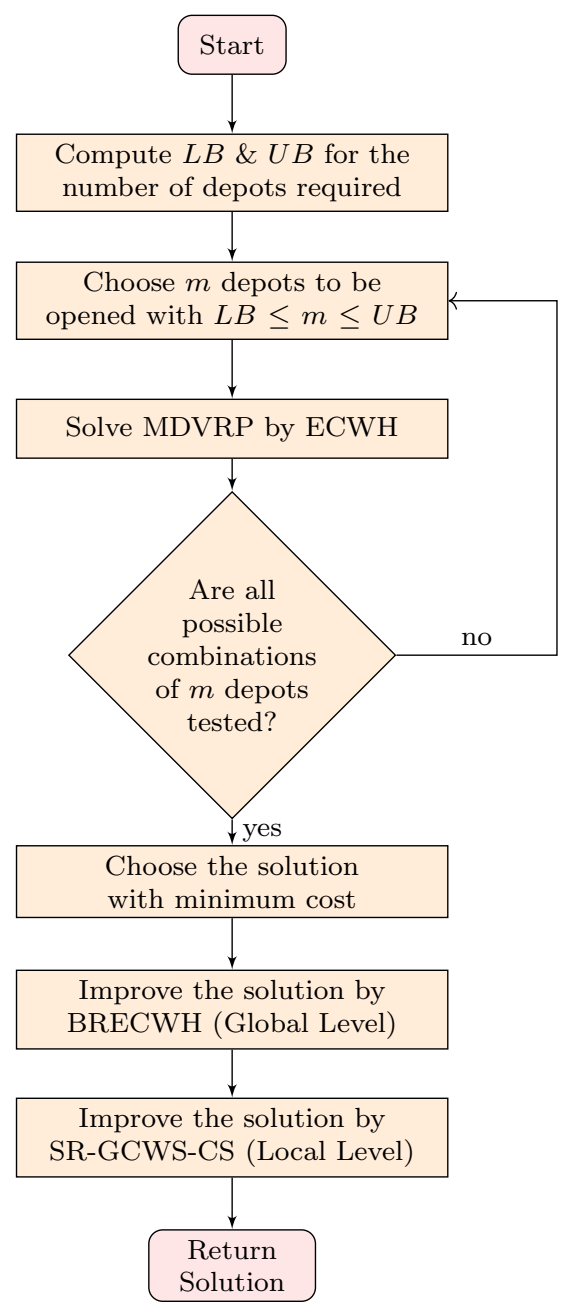

Fig. 2 The multi-start biased-randomized heuristic for the LRPCD

1. The combined demand of the new route should not exceed the vehicle loading capacity.

2. Before the merging process, customers $i$ and $j$ must not be on the same route.

3. If a customer is directly connected to two other customers, then it is never considered for linking.

4. The distance constraint must be satisfied.

If one or more of the conditions are not satisfied, this pair of customers is excluded from further being served by the current depot, and they are considered to be served by other depots. If all the conditions are satisfied, then the customers are served by the current depot and are eliminated from 
consideration at any other depot. The ECWH proposed by Tillman $(1969)$ is similar to the classical savings heuristic proposed by Clarke and Wright (1964). However, the difference between mentioned heuristics is in the calculation of the savings and the fact that the former updates the savings values after each iteration.

\subsection{Second Stage: Improvement of Promising Solutions}

The improvement stage consists of two levels to reallocate customers and improve the routing for the best solution found in the first stage. These two levels are called global level and local level. In the global level, the customer reallocation and routing is improved for the best solution found in the first stage by means of a biased-randomized version of the ECWH (BRECWH). In the BRECWH, the ECWH savings list is randomized using the geometric probability distribution to generate a different solution each time it is run. For the LRP, this procedure generates solutions which are already relatively close to the best-known solution (BKS) in the literature. In the local level, to improve the routing decisions, we apply the SR-GCWS-CS algorithm described in Juan et al (2011b). The SR-GCWS-CS combines the biased randomized technique with the classical savings heuristic proposed by Clarke and Wright (1964) for the VRP.

\section{A Biased-Randomized VNS Meta-heuristic}

In order to generate higher-quality solutions for the LRPCD, we propose a Biased-Randomized VNS (BR-VNS) meta-heuristic, as shown in Algorithm 1 . Customer nodes are allocated to depots according to the savings value, $\mu_{i j}$, associated with serving customer $j$ from depot $i$. The value $\mu_{i j}$ is defined as the saving resulted from the cost difference between serving customer $j$ from depot $i$ and serving customer $j$ from its best-alternative depot, $i^{*}$, i.e., $\mu_{i j}=c_{i j}-$ $c_{i^{*} j}$. Once the savings list has been created for each depot, the customers-todepots allocation mapping is constructed using a round-robin process. During this process, biased randomization techniques are employed so that different promising mappings are quickly generated each time the procedure is run. Thus, at each turn a depot chooses its next customer according to a geometric distribution with parameter $\beta$, as proposed by (Juan et al, 2015).

Furthermore, the planning of the delivery routes also uses biased randomization techniques. In particular, we use the same biased-randomized version of the classical savings heuristic that was proposed for the multi-start heuristic.

During the construction of feasible solutions, the upper and lower bounds $(U B / L B)$ concerning the number of depots to be opened are computed under the consideration of overall customers' demand and depots' capacities. Subsequently, different random combinations of $m$ depots $(L B \leq m \leq U B)$ are generated. The customer allocations and delivery route planning are then optimized during nInitialIters iterations. From the initial solutions, the nPromising 


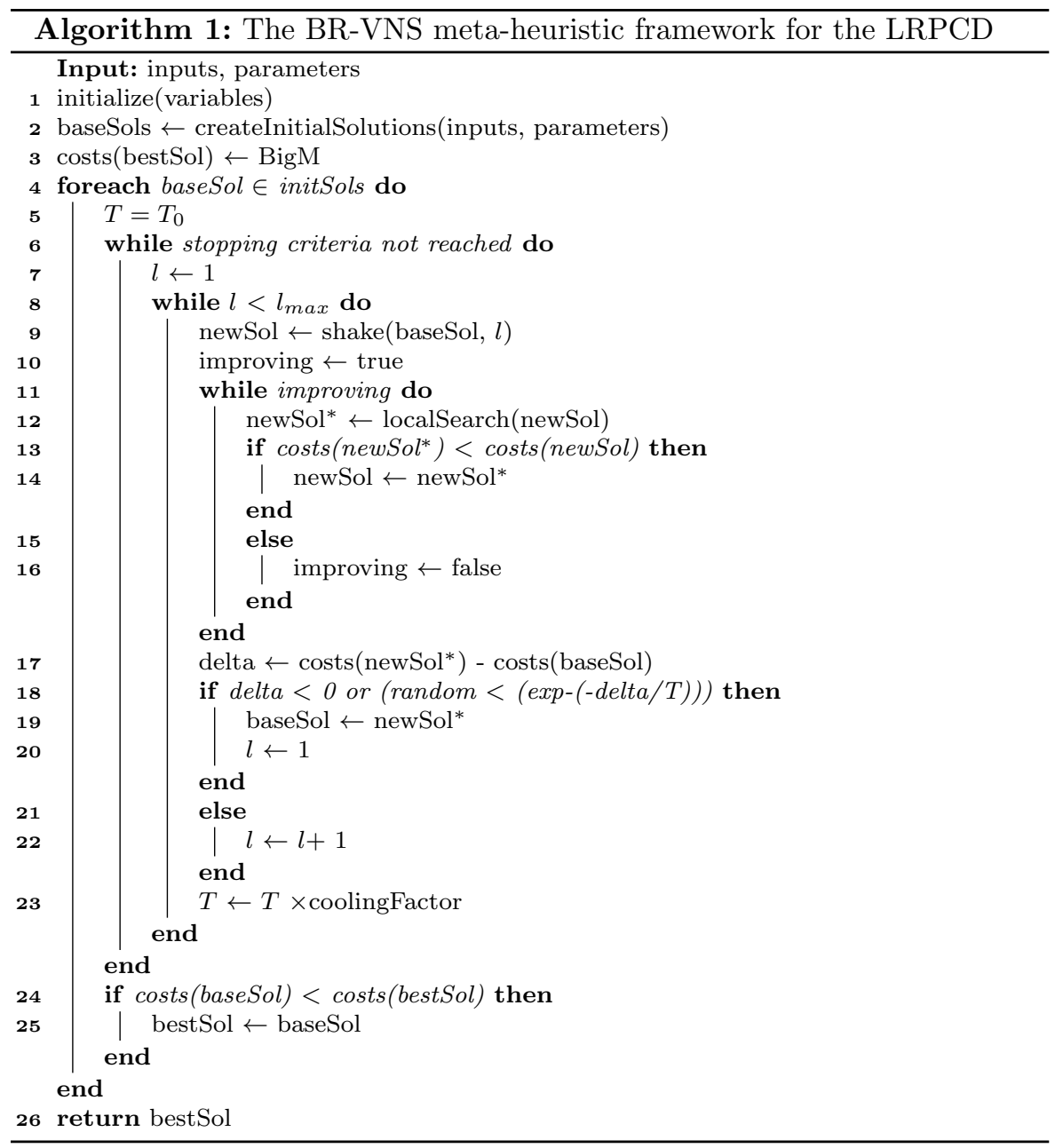

most promising ones are stored within a set of baseSols. Each potential solution is then further improved through a VNS metaheuristic framework.

The BR-VNS framework is based on construction of different solution neighborhoods, which are then passed through a given local search operator. For any solution baseSol $\in$ baseSols, different shaking procedures are executed to alter the current solution and obtain different neighborhood structures $N_{l}$ $\left(l=1,2, \ldots, l_{\max }\right)$. The shaking procedure consists of randomly exchanging the depot allocation of $\% p$ of all customers. Furthermore, the percentage values applied at this point are increasingly taken from the range $p=0.05,0.10, \ldots, 0.95$.

After the structure of each baseSol has been changed to create a new solution newSol, a local search is applied to find the local minimum within the current neighborhood solution. We have designed three different local search 
operators, which are depicted in Table 1. In each iteration, one of these local search operators is randomly chosen.

Table 1 Local Search Operators

\begin{tabular}{ll}
\hline Operator (k) & Description \\
\hline Customer Swap Inter-Route & Swaps customers randomly chosen between \\
& different routes of the same depot. \\
Inter-Depot Node Exchange & Exchanges two nodes randomly selected \\
& from different depots. \\
& Interchanges positions of 3 random, non-consecutive \\
Cross-Exchange & customers from different depots. \\
\hline
\end{tabular}

A newSol is accepted as the new baseSol if the associated cost of the former outperforms that of the latter. Moreover, we also apply a simulated annealing-like acceptance criterion for non-improving solutions, which uses an initial temperature $T_{0}$ and a cooling constant coolingFactor as described by Henderson et al (2003). Finally, the current bestSol is updated whenever it is outperformed by the newSol. This procedure is repeated until a predefined stopping criterion (maxIter) is reached. Then, the best-found solution is returned to the decision maker.

\section{Computational Experiments}

A series of computational experiments have been conducted in order to evaluate the performance of the proposed solution methods both for the LRP and the LRPCD. The proposed MSBRH heuristic and BR-VNS meta-heuristic have been coded as Java applications. All experiments have been performed using a $2.3 \mathrm{Ghz}$ Quad-Core AMD Opteron(tm) processor with 8 GB of RAM and running under CentOS release 6.6. We have used three classical benchmark sets for the LRP. The first benchmark set is known as the Barretos's set (Barreto, 2004). It contains a total of 17 instances with 2 to 15 possible depot locations and the number of customers ranging from 12 to 150 . The second set is known as the Akca's set (Akca et al, 2009). It includes 12 instances with 5 depots and 30-40 customers. The third set is known as the Prodhon's set (Belenguer et al, 2011). It involves a total of 30 instances ranging from 5-10 potential logistics facilities and 20-200 customers.

Since there are no specific benchmarks for the LRPCD, we have generated new instances based on the LRP ones. In order to facilitate the comparison, we have adapted all instances as follows: (i) we select a random sample of 10 instances from each benchmark set; (ii) each of these instances is solved using the BR-VNS metaheuristic; (iii) route distances from each solution are sorted according to their distances; (iv) we select, for each set, the route length corresponding to the 3rd quartile of them; and $(v)$ this value is rounded to the nearest multiple of 10 . As a result, the distance is constrained with values of 5,500 and 130 for Prodhon's and Akca's sets, respectively. Regarding 
Barreto's instances, we could not achieve a single value for the constrained distance. Therefore, distance constraint values are 700 for both Min-27x5 and Min92-134x8 instances, 3,960 for Daskin95-150x10, and 130 for the remaining instances of this set. During the computational experiments, each instance has been run using 10 different random seeds, and the best result is considered as our best solution $(B S)$. According to some preliminary tests, the following parameters have been chosen for the computational experiments.

- Number of iterations for initial solution: nInitialIters $=500$.

- Number of promising solutions: nbaseSols $=2+\lfloor$ nodes $/ 100\rfloor$.

- Stopping criterion $(\operatorname{maxIter})=350$.

- Iterations for BR savings heuristic: routingIterationsRandomCWS= 150 .

- Geometric parameter for BR savings heuristic: $0.07 \leq \alpha \leq 0.23$.

- Geometric parameter for BR allocation mapping: $0.05 \leq \beta \leq 0.8$.

- Initial SA temperature: $T_{0}=100$.

- SA cooling constant: coolingFactor $=0.994$.

In the remaining of this section, we discuss the results obtained by our two approaches (MSBRH and BR-VNS) when tested in two different scenarios: (i) the standard LRP - which is used to compare our methods against the BKS provided in the literature; and (ii) the LRPCD - in order to illustrate the potential of our methods. We need to emphasize that we offer two types of solution methods to the decision maker, namely: the fast MSBRH and the BR-VNS meta-heuristic. We report on their performance comparison in order to illustrate that each method has its pros and cons when applied in a business context. The MSBRH offers good-quality solutions in just a few seconds, which makes it favorable in an operational level when a quick solution is required. However, the metaheuritic provides higher-quality solutions at the expense of computational time.

\subsection{Experimental Analysis for the Standard LRP}

The computational experiments for the standard LRP are shown in Tables 2 3 , and 4, which compare the results of the proposed MSBRH and BR-VNS algorithms with the best-known solutions (BKS) reported in the literature for Barreto, Akca, and Prodhon's benchmarks.The first, second, and third column in each table shows the instance name, the BKS cost, and the corresponding computational time to obtain the solution. The following columns show the average cost obtained using each solution method for different runs (Average Total Cost), the best solution found (BS Total Cost), its associated opening cost (BS Opening Cost), distance-based cost (BS Distance), and (BS Vehicles). This last value corresponds to the number of vehicles in Barreto's and Akca's sets, while it represents the vehicle cost in Prodhon's set. The following columns are: $(B S G A P)$ for the percentage gap of BS values with respect to the BKS calculated as $100[(B S-B K S) / B K S]$; and (BS CPU (sec)) for com- 
putational times in seconds. Whenever our best-found solution matches the BKS in the literature, the corresponding value has been indicated in boldface.

Considering BS Total Cost columns in Table 2, two general trends for small and large instances can be observed. In smaller instances with up to 36 customers, MSBRH tend to perform as well as BR-VNS, and it can even match $B K S$ with the only exception of Gaskell $-32 x 5$, where the gap is $3.66 \%$. In larger instances with more than 36 customers, BR-VNS outperfoms MSBRH. MSBRH has a considerably good performance on Barreto's instances compared to the other two benchmarks, and can match, employing just a few seconds, 7 out of 17 BKS results in the smallest instances. BR-VNS can also obtain the BKS result in 5 out of 17 instances.

As reported in Table 2, in larger instances with more than 36 customers BR-VNS obtains better results compared to MSBRH - although the former requires also larger computational times. However, there are two exceptions: in Christ $-75 \times 10$ and Perl $83-85 x 7$ instances, MSBRH outperforms BRVNS. In general, the average computational time employed by MSBRH is 3.88 seconds, while its average gap is $1.59 \%$. Meanwhile, BR-VNS shows an average computational time of 99.8 seconds, but its average gap with respect to BKS is down to $0.72 \%$. Moreover, in both approaches, the average computational time improves the computational time required to obtain the BKS.

The results in Table 3 confirm our previous observation in Barreto's set about the direct effect of the instance size on the performance of both solution methods. Akca's instances include either 30 or 40 customers. As expected in larger instances, BR-VNS obtains better solutions in terms of best-found total cost and average total cost. BR-VNS can effectively solve Akca's instances and match BKS in 7 out of 12 instances, whereas MSBRH can reach only 3 out of 12 BKS results. Therefore, BR-VNS performs better than MSBRH in terms of both solution quality and computational time.

BR-VNS outperforms MSBRH in most of Prodhon's instances (Table 4). There are two exceptions: coord $20 x 5-1 b$ and coord $20 x 5-2 b$, where both BR-VNS and MSBRH can match the BKS. In smaller instances with up to 50 customers and 5 depots (e.g., instance coord50x5-2), BR-VNS performs very well and can usually match the BKS result.

In general, BR-VNS tends to outperform the simpler MSBRH, although the latter is able to offer quite competitive solutions in almost real time, which is illustrated in Figure 3, which presents a multiple box plot comparison of MSBRH and BR-VNS for the standard LRP with no distance constraints for three benchmark sets.

\subsection{Experimental Analysis for the LRPCD}

In order to provide an objective comparison of the results obtained by means of our both procedures, we have compared them against the results obtained by exact methods. To do so, we have coded the LRPCD using GAMS modeling language and used both CPLEX and Gurobi as solvers. All experiments 


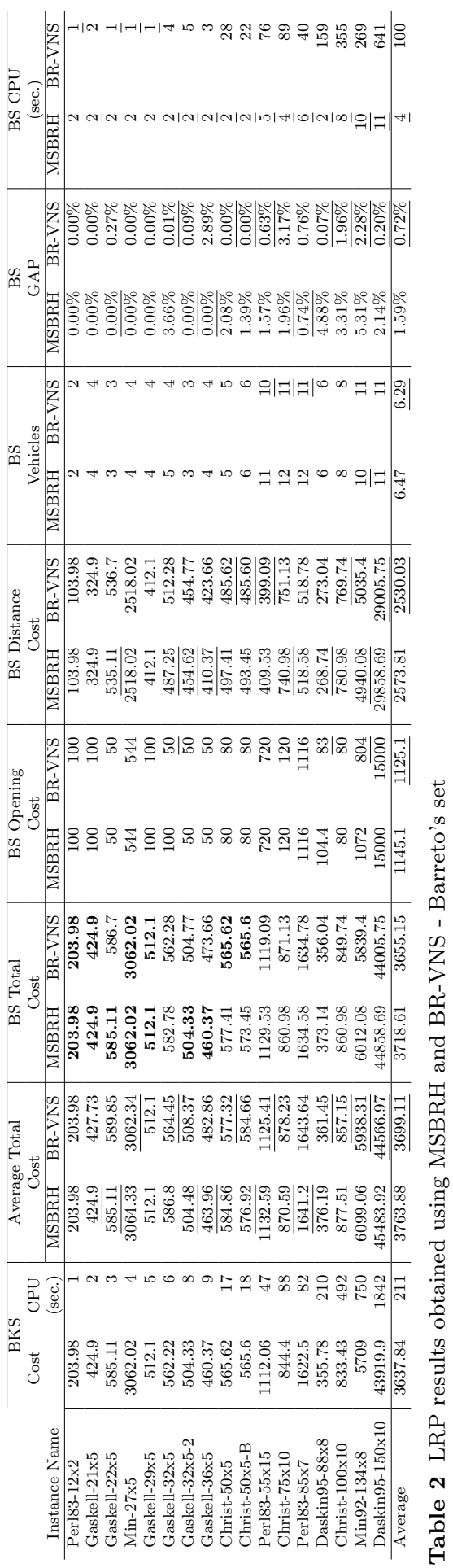




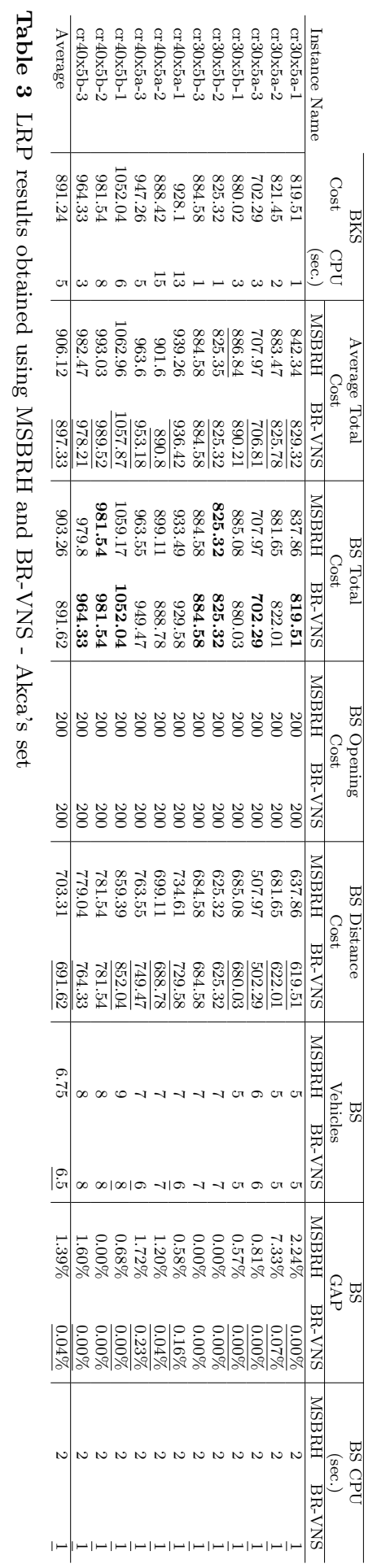




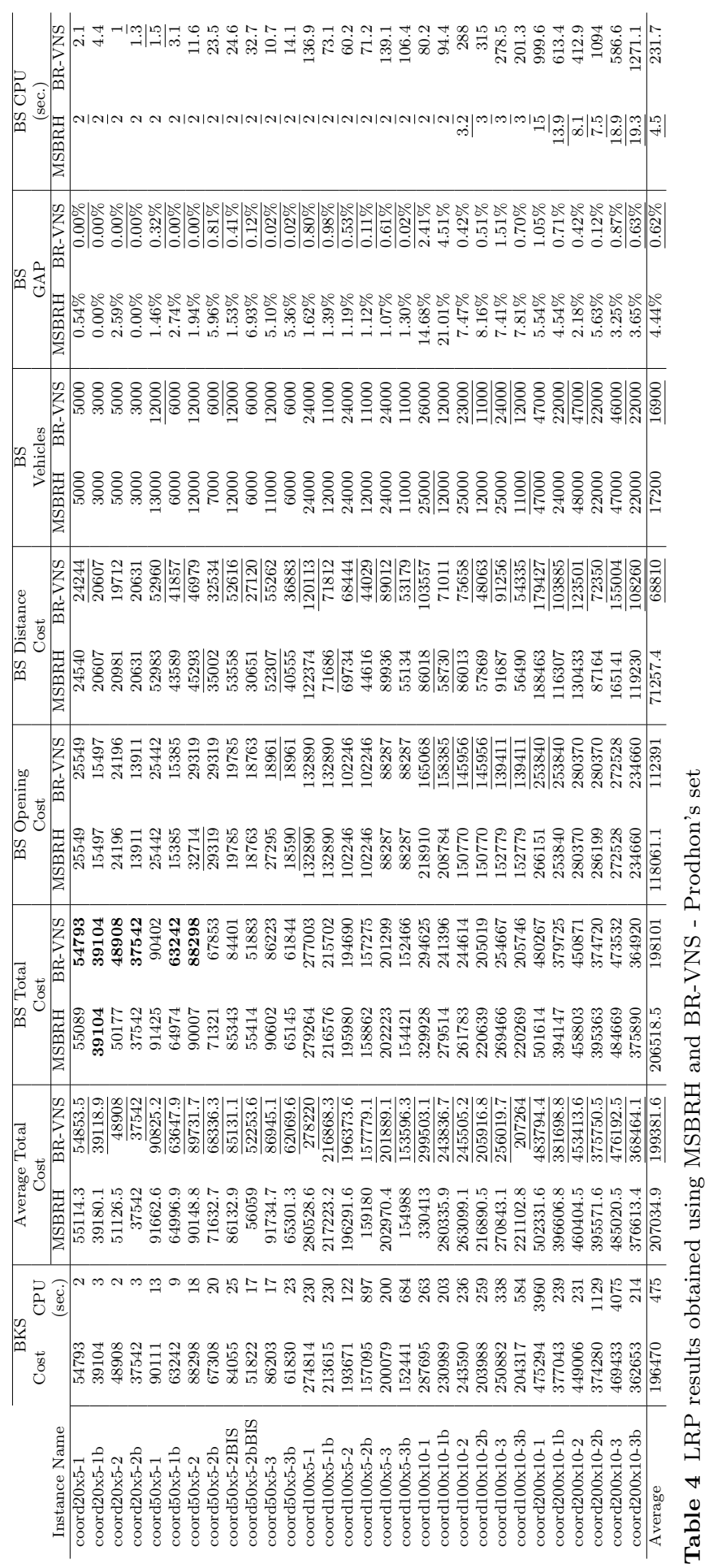




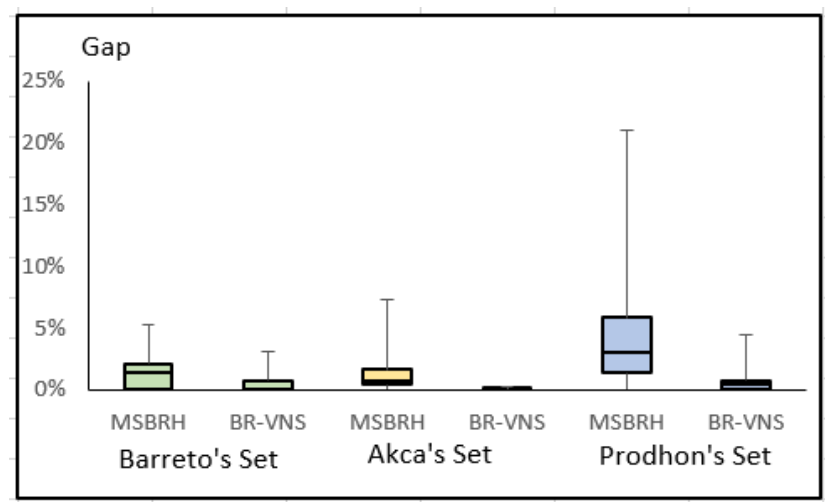

Fig. 3 The average \%gap of the BS Total Cost wrt BKS for LRP

were uploaded to Neos Solvers (http://neos-server.org) with 27000 secs (i.e. 7.5 hours) of maximal execution time.

In Table 5 we compare our results against the best solution obtained by GAMS Cplex/Gurobi solvers for the three benchmark sets. It is important to notice that: (i) For instances with more than 40 customers, the solvers run out of memory and did not provide any solution; (ii) None of the solutions reported by the solvers was proven to be optimal; (iii) Cplex was not able to solve instances from Akca's set. It can be seen that, regarding Barreto's set, the average gap of our approximate methods are $2.97 \%$ and -0.03 for the heuristic and metaheuristic, respectively. Regarding Akca's set, GUROBI was able to solve 9 out of 12 instances. In average, both approximate methods outperform the exact one with gaps of $-1.25 \%$ and $-5.16 \%$ for the heuristic and the metaheuristic, respectively. With respect to Prodhon's instances, GUROBI only provided results for 3 instances. The average gap calculated over such instances is $0.56 \%$ and $-0.76 \%$ for the heuristic and metaheuristic, respectively. The global average gap shows the competitiveness of both approaches compared to the exact method. Gap values are $0.19 \%$ and $-2.98 \%$ for MSBRH and BR-VNS, respectively.

In addition, Tables 6, 7, and 8 present the detailed results provided by MSBRH and BR-VNS for the LRPCD. The following information is gathered in these tables: instance name, BKS, (Average Total Cost) for different runs, best-found solution (BS Total Cost), opening cost (BS Opening Cost), distance-based cost (BS Distance Cost), and (BS Vehicles). This column corresponds to the number of vehicles in Barreto's and Akca's sets, while it reflects on the cost of vehicles in the Prodhon's set. The last columns correspond to the associated gaps, and computational times in seconds.

In Table 6, we can observe that BR-VNS noticeably outperforms MSBRH in terms of solution quality in 14 out of 17 instances. Among the other 3 instances, MSBRH can match BR-VNS in 2 instances, and it outperforms BR-VNS in 1 instance. This is due to the effectiveness of BR-VNS in routing decisions. In terms of computational time, BR-VNS is usually faster in smaller 


\begin{tabular}{|c|c|c|c|c|c|}
\hline Instance Name & Gurobi(1) & MSBRH(2) & BR-VNS(3) & GAP(2) - (1) & GAP(3) - (1) \\
\hline Perl-12x2 & 203.98 & 203.98 & 203.98 & $0.00 \%$ & $0.00 \%$ \\
\hline Gas-21x5 & 430.14 & 465.73 & 424.9 & $8.27 \%$ & $-1.22 \%$ \\
\hline Gas-22x5 & 775.12 & 789.74 & 775.12 & $1.89 \%$ & $0.00 \%$ \\
\hline Min-27x5 & 3770.98 & 3770.98 & 3770.98 & $0.00 \%$ & $0.00 \%$ \\
\hline \multicolumn{7}{|c|}{ Average GAP Barreto's Set } \\
\hline Cr30x5a-1 & 977.32 & 892.4 & 892.4 & $-8.69 \%$ & $-0.03 \%$ \\
\hline Cr30x5a-2 & 915.38 & 1006.65 & 915.54 & $9.97 \%$ & $0.02 \%$ \\
\hline Cr30x5a-3 & 704.66 & 707.97 & 702.29 & $0.47 \%$ & $-0.34 \%$ \\
\hline Cr30x5b-1 & 952.83 & 1098.63 & 952.83 & $15.30 \%$ & $0.00 \%$ \\
\hline Cr30x5b-2 & 922.22 & 971.19 & 922.65 & $5.31 \%$ & $0.05 \%$ \\
\hline Cr40x5a-1 & 1098.13 & 979.78 & 979.42 & $-10.78 \%$ & $-10.81 \%$ \\
\hline Cr40x5a-2 & 1017.36 & 913.25 & 899.69 & $-10.23 \%$ & $-11.57 \%$ \\
\hline Cr40x5a-3 & 1061.94 & 1009.94 & 985.36 & $-4.90 \%$ & $-7.21 \%$ \\
\hline Cr40x5b-3 & 1053.38 & 993.23 & 989.55 & $-5.71 \%$ & $-6.06 \%$ \\
\hline \multicolumn{7}{|c|}{ Average GAP Akca's Set } \\
\hline Coord20-5-1 & 55793 & 55806 & 55806 & $0.02 \%$ & $0.02 \%$ \\
\hline Coord20-5-2 & 50918 & 51960 & 49931 & $2.04 \%$ & $-1.93 \%$ \\
\hline Coord20-5-2b & 54715 & 54729 & 54742 & $0.03 \%$ & $0.05 \%$ \\
\hline \multicolumn{7}{|c|}{ Average GAP Prodhon's Set } \\
\hline
\end{tabular}

Table 5 Comparison of our LRPCD results against the ones obtained by an exact method

instances with up to 29 customers, while MSBRH shows considerably lower computational times in larger instances with more than 29 customers. Considering the computational times and the average gap of $-4.10 \%$, we can claim that BR-VNS is a viable alternative for strategic decisions, while MSBRH is still competitive when a quick solution is required. Table 7 results illustrate that BR-VNS performs considerably better than MSBRH in terms of both solution quality and computational time. BR-VNS outperforms MSBRH in 9 out of 12 instances, and it matches MSBRH results in the other 3 instances. In Akca's set, the number of customers is either 30 or 40, which indicates the instances are not very large. In Table 8, BR-VNS outperforms MSBRH in 24 out of 30 instances. This confirms the trend that we have reported on large instances of Barreto's set.

To investigate the effect of the distance constraints, we compute the percentage gap between LRP and LRPCD for BS Opening Cost as \%gap = $100\left[\left(\right.\right.$ opening cost $_{L R P}-$ opening cost $\left._{L R P C D}\right) /$ opening $\left.\operatorname{cost}_{L R P}\right]$. A similar formula is applied for the BS Distance Cost and BS Vehicle. Table 9 summarizes the results and Figures 4. 5, and 6 compare the average percentage gap obtained by MSBRH and BR-VNS. As expected, the average gaps for BS Opening Cost, BS Distance Cost, and BS Vehicle have increased in most benchmark sets after applying the distance constraint. Notice that BR-VNS can deal with the addition of the distance constraint better than MSBRH. However, the percentage average gaps of MSBRH and BR-VNS are not massively different and, more interestingly, the values follow the same pattern; for instance, both solution methods produce $45.13 \%$ and $41.10 \%$ (the highest values of BS Vehicles in Prodhon's set), or $6.43 \%$ and $3.16 \%$ (the lowest values of BS Distance Cost in Barreto's set). This fact shows that the characteristics of the benchmark 


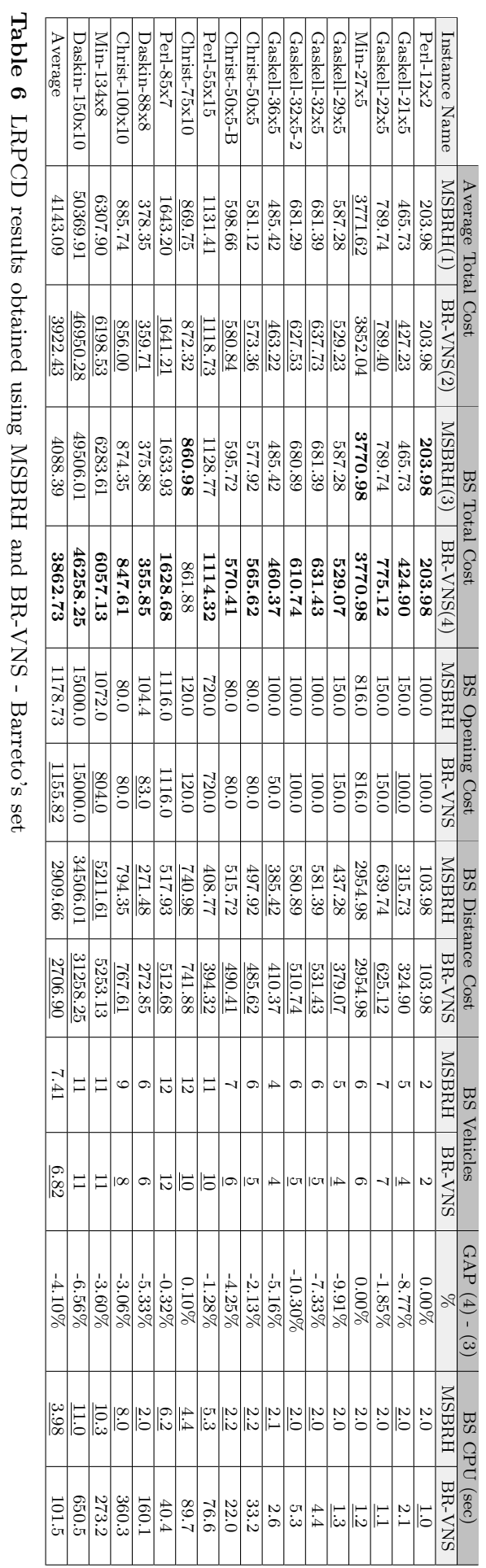




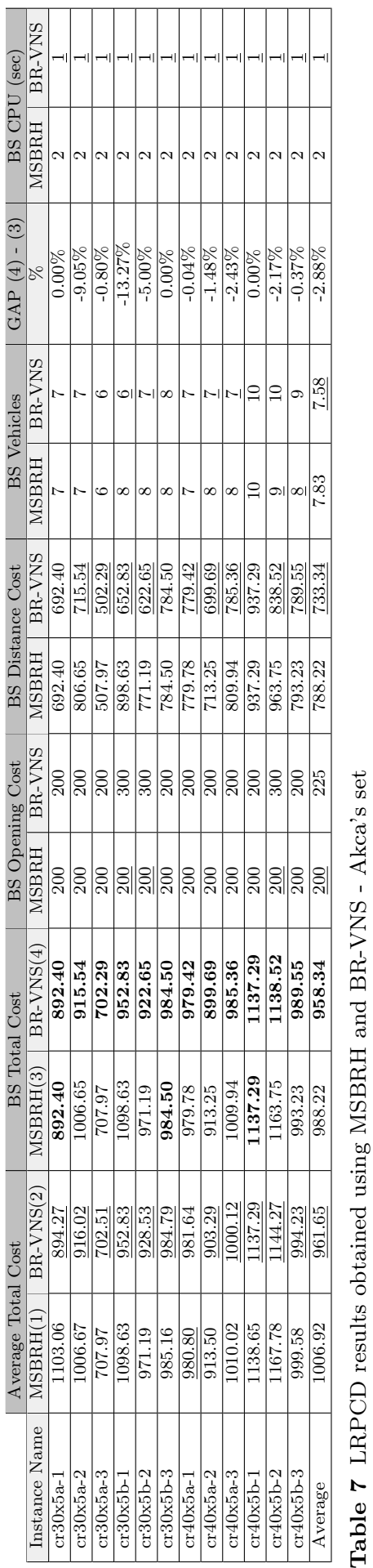




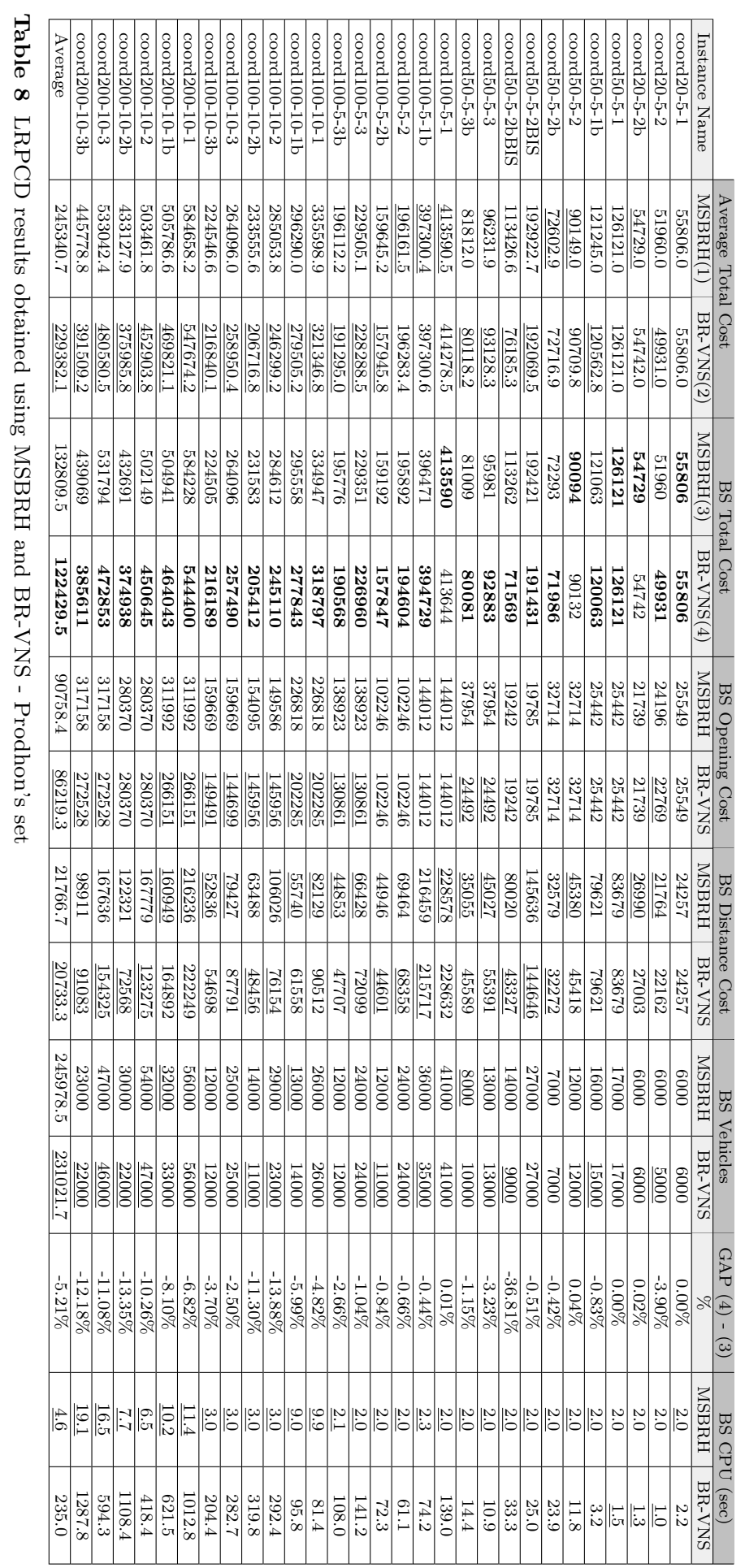


sets play an important role in the final LRP and LRPCD solutions. We can conclude that the introduction of the distance constraint leads to a noticeable increase in all costs. In other words, employing EVs with limited driving ranges can potentially increase the total distribution cost.

\begin{tabular}{|l|c|c|c|c|c|c|}
\multicolumn{5}{c|}{ MSBRH } & \multicolumn{3}{c|}{ BR-VNS } \\
\hline Data Set & BS Opening Cost & BS Distance Cost & BS Vehicle & BS Opening Cost & BS Distance Cost & BS Vehicle \\
\hline Barreto's set & $32.35 \%$ & $6.43 \%$ & $24.26 \%$ & $29.41 \%$ & $3.16 \%$ & $17.80 \%$ \\
\hline Akca's set & $0.00 \%$ & $12.04 \%$ & $18.40 \%$ & $12.50 \%$ & $5.90 \%$ & $17.51 \%$ \\
\hline Prodhon's set & 17.43 & $33.72 \%$ & $45.13 \%$ & $13.34 \%$ & $31.05 \%$ & $41.10 \%$ \\
\hline
\end{tabular}

Table 9 The average gap between LRPCD and LRP

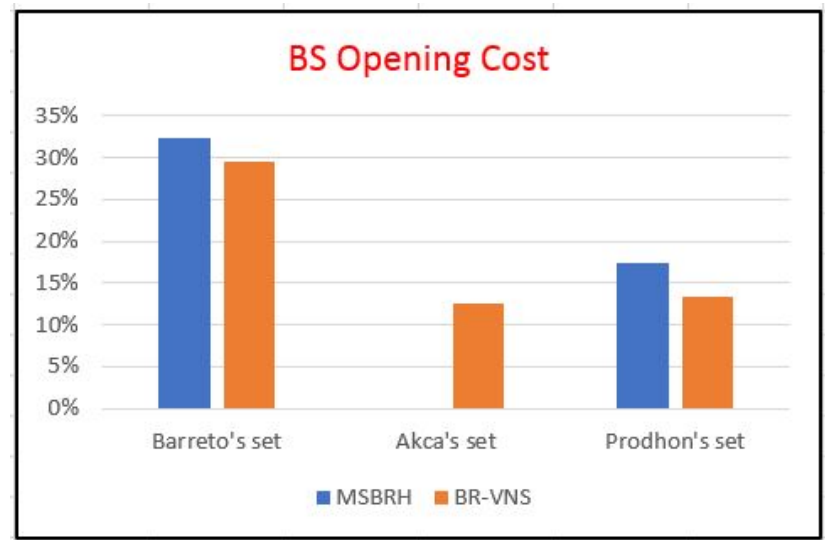

Fig. 4 The average \%gap of the Opening Cost for LRPCD

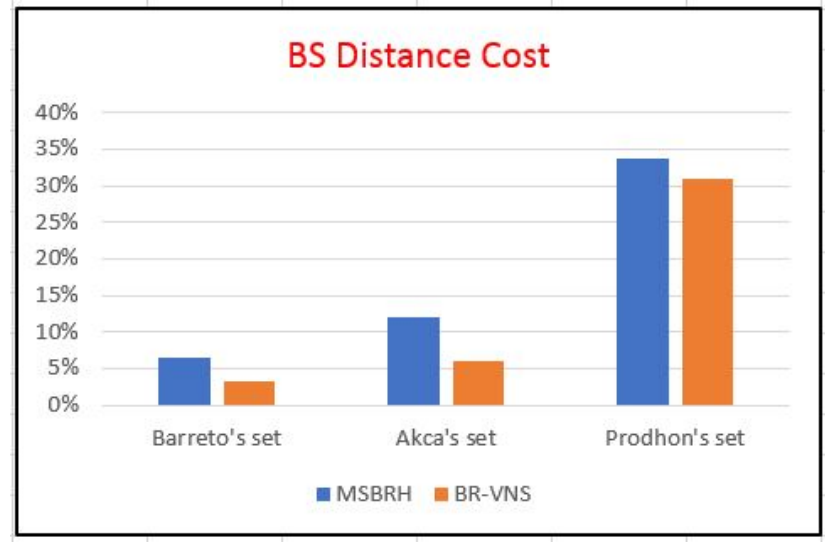

Fig. 5 The average \%gap of the Distance Cost for LRPCD 


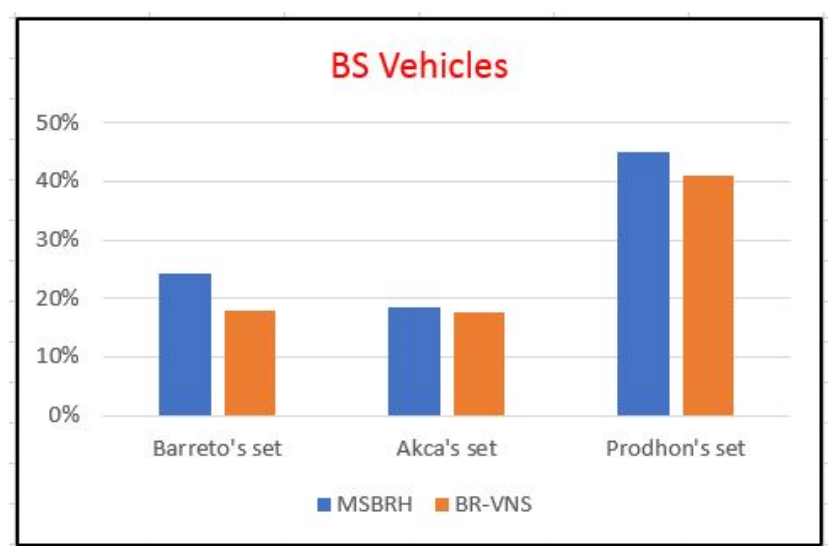

Fig. 6 The average \%gap of the Vehicles for LRPCD

\subsection{Sensitivity Analysis}

Due to the importance of the distance constraint in EVs, a sensitivity analysis has been conducted to investigate the effect of maximum distance allowed for each vehicle on the opening cost, the variable distance cost, and the fixed cost of using vehicles. To perform the sensitivity analysis, we have considered Barreto's and Prodhon's sets as they are comprised of small, medium and large size instances. Akca's set is not considered in the sensitivity analysis as the instance sizes are limited to only small and medium. We have randomly selected one instance from each category of small, medium and large-sized instances from each of Barreto's and Prodhon's sets. Therefore, we have a total of 6 different instances which are shown in Tables 10 and 11 where the results of MSBRH and BR-VNS are presented, respectively.

The sensitivity analysis is designed based on the assumption that the maximum distance allowed for vehicles, $\bar{d}$, is equal to 130 for Barreto's instances and 5500 for Prodhon's instances as mentioned in Section 6. In the test experiments, we have noticed that if the maximum distance is reduced by $10 \%$ or more, the solution will be infeasible. Hence, we set the experiments in the feasible range and run experiments for instances with $\bar{d}-3 \% \bar{d}$, $\bar{d}-6 \% \bar{d}$ and $\bar{d}-9 \% \bar{d}$ as shown in Tables 10 and 11 . Therefore, performing experiments on 6 instances with 3 different maximum driving ranges and applying MSBRH and BR-VNS means that we have a total number of 36 instances in the sensitivity analysis. To investigate the effect of the maximum distance, each instance has been run 10 times with different random seeds and the best solution is reported as a percentage deviation with respect to the current solution for $\bar{d}$. For instance, we compute the deviation percentage for BS Opening Cost for $\bar{d}-3 \% \bar{d}$ in Table 10 as \%deviation = $100\left[\left(\right.\right.$ opening $\operatorname{cost}_{\bar{d}-3 \% \bar{d}}-$ opening $\left.\operatorname{cost}_{\bar{d}}\right) /$ opening cost $\left._{\bar{d}}\right]$. Similar calculation is 
performed for the BS Distance Cost, BS Vehicle, and BS Total Cost in both Tables. Whenever there is an increase in cost and the \% deviation value is positive, the corresponding value has been indicated in boldface.

As expected, we can observe that the BS Opening Cost obtained by MS$\mathrm{BRH}$ in Table 10, is not affected by changing maximum distance in any instance other than coord $20 x 5-2$, where the percentage deviation of -5.9 shows opening cost decreases with the highest decrease in maximum distance that is $\bar{d}-9 \% \bar{d}$. Although the same pattern of no effect on opening cost is true for randomly selected Barreto's instances when BR-VNS is applied in Table 11. the opening cost of almost all of the Prodhon's instances are increased with an exception of opening cost of coord $20 x 5-2$ which remains unchanged. In average, the BS Opening Cost percentage deviations using MSBRH is 0.0 other than the average value of -1.0 for $\bar{d}-9 \% \bar{d}$ which is due to unusual behaviour of coord $20 x 5-2$. However, the average of BS Opening Cost percentage deviations applying BR-VNS is 10.0 which indicates an overall increase for all different maximum driving ranges.

Another interesting finding in Table 10 is that by decreasing the maximum distance, the $B S$ Distance Cost values increase in all instances other than Gaskell-21x5, where the cost is unchanged. However, the BS Vehicles values are reduced in 3 instances Christ $-50 x 5-B$, Perl $83-85 x 7$ and coord $100 x 10-$ 1 , and they remain the same in the other 3 instances. The only exception is coord $20 x 5-2$, where both routing and vehicle costs increase when the maximum limit is equal to $\bar{d}-9 \% \bar{d}$. This means that in general, lower maximum driving ranges can result in higher routing costs in almost all instances and half of the instances may need less number of vehicles, especially when the instance size is larger. Corresponding positive average values of routing costs and negative values of vehicles costs also reflect this observation.

On the other hand, BR-VNS results in Table 11. show two different patterns for Barreto's and Prodhon's set. In Barreto's set, more limited driving range leads to either increase or no change in routing costs and no change in vehicle costs. Instead in Prodhon's set, by reducing maximum driving range, the routing cost becomes less in all instances, but the vehicle cost can decrease, stay the same or even increase in different instances as shown in Table 11. This different effect on Barreto's and Prodhon's set can be explained due to the important role of the benchmark characteristics, which is well in line with our previous findings for the LRPCD in Section 6.2 Overall, the average percentage deviations of routing costs are decreased and the average percentage deviations of vehicles costs are increased other than for the smallest maximum driving range of $\bar{d}-3 \% \bar{d}$.

It is noticeable that $B S$ Total Cost increases by adding more limitations to driving range in 32 out of 36 instances, which is reflected in the positive average percentage deviations. This confirms our conclusion in Section 6.2 for the effect of driving range on overall cost results provided by MSBRH and BR-VNS on LRPCD. 


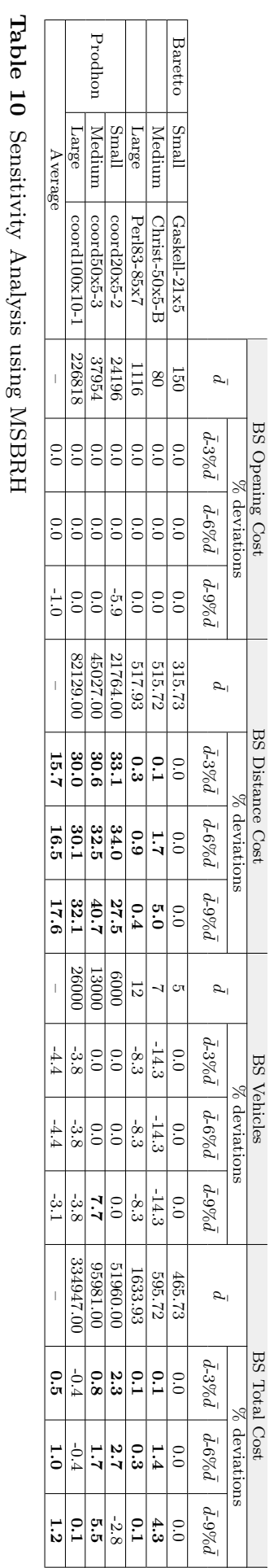




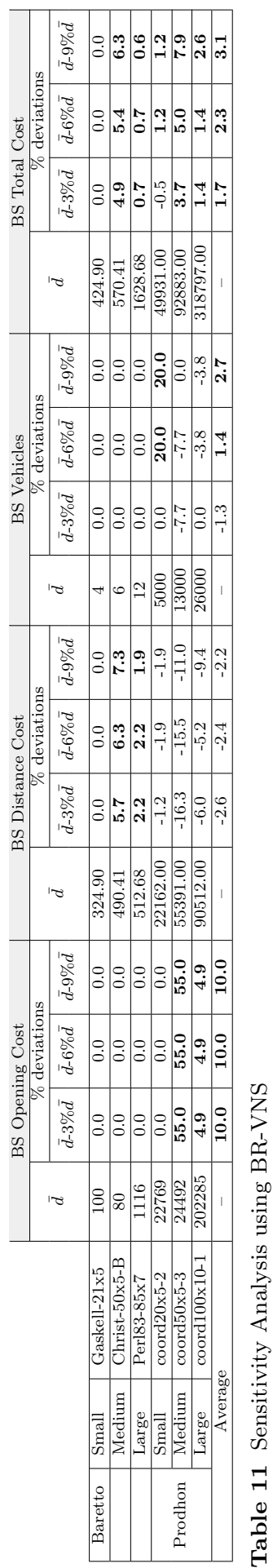




\section{Conclusions and Future Research}

This research study discusses the Location Routing Problem with a Constrained Distance (LRPCD), which considers the use of electrical vehicles with limited driving ranges. The use of electric vehicles is gaining interest in the delivery fleets, since it allows for greener transportation activities. To the best of our knowledge, this is the first time that this realistic extension of the LRP has been studied.

To solve the LRPCD problem, we propose two approaches:, a multi-start biased-randomized heuristic (MSBRH) and a biased-randomized Variable Neighborhood Search (BR-VNS) metaheuristic. A series of computational experiments have been conducted to evaluate the performance of these methods in solving benchmark instances for the classical LRP problem as well as for newly generated LRPCD instances. The experimental results show that MSBRH is a fast approach and is able to generate good-quality solutions in almost realtime, which makes it useful in some situations in which decisions need to be made very quickly (e.g., in some telecommunication systems). Additionally, $\mathrm{BR}-\mathrm{VNS}$ is able to provide even better solutions with higher computational times.

As future research work, we would like to consider richer versions of the LRPCD by adding stochastic travel times as well as heterogeneous fleets of vehicles - in terms of loading capacity. Also, it would be interesting to investigate different fleet configurations and analyze the trade-off between how much "green" is each configuration and its associated distance-based cost. It should be noted that configurations employing "greener" vehicles might require more routes and, therefore, larger distances.

\section{Acknowledgments}

This work has been partially supported by the Spanish Ministry of Economy and Competitiveness and FEDER (TRA2015-71883-REDT), the Erasmus+ program (2018-1-ES01-KA103-049767), and the UoP doctoral programs.

\section{References}

Achtnicht M, Bühler G, Hermeling C (2012) The impact of fuel availability on demand for alternative-fuel vehicles. Transportation Research Part D: Transport and Environment 17(3):262-269

Ahn J, de Weck O, Geng Y, Klabjan D (2012) Column generation based heuristics for a generalized location routing problem with profits arising in space exploration. European Journal of Operational Research 223(1):47-59

Akca Z, Berger RT, Ralphs TK (2009) A Branch-and-Price Algorithm for Combined Location and Routing Problems Under Capacity Restrictions, Springer US, Boston, MA, pp 309-330 
Aksen D, Altinkemer K (2008) A location-routing problem for the conversion to the "click-and-mortar" retailing: The static case. European Journal of Operational Research 186(2):554-575

Barreto S (2004) Analysis and modelling of location-routing problems. $\mathrm{PhD}$ thesis, University of Aveiro, Portugal

Bektaş T, Demir E, Laporte G (2016) Green Transportation Logistics, Springer International Publishing, chap Green vehicle routing, pp 243-265

Belenguer JM, Benavent E, Prins C, Prodhon C, Calvo RW (2011) A branchand-cut method for the capacitated location-routing problem. Computers \& Operations Research 38(6):931-941

Caballero R, González M, Guerrero FM, Molina J, Paralera C (2007) Solving a multiobjective location routing problem with a metaheuristic based on tabu search. application to a real case in andalusia. European Journal of Operational Research 177(3):1751-1763

Chan CC, Wong YS, Bouscayrol A, Chen K (2009) Powering sustainable mobility: Roadmaps of electric, hybrid, and fuel cell vehicles [point of view]. Proceedings of the IEEE 97(4):603-607

Chan Y, Baker SF (2005) The multiple depot, multiple traveling salesmen facility-location problem: Vehicle range, service frequency, and heuristic implementations. Mathematical and Computer Modelling 41(8-9):1035-1053

Chellaiah C, R D (2017) Future renewable energy option for recharging full electric vehicles. Renewable and Sustainable Energy Reviews 76:824-838, DOI 10.1016/j.rser.2017.03.032

Clarke G, Wright J (1964) Scheduling of vehicles from a central depot to a number of delivery points. Operations Research 12:568-581

Coutinho-Rodrigues J, Tralhão L, Alçada-Almeida L (2012) Solving a locationrouting problem with a multiobjective approach: the design of urban evacuation plans. Journal of Transport Geography 22:206-218

Crainic TG (2000) Service network design in freight transportation. European Journal of Operational Research 122(2):272-288

De Armas J, Juan AA, Marquès JM, Pedroso JP (2017) Solving the deterministic and stochastic uncapacitated facility location problem: from a heuristic to a simheuristic. Journal of the Operational Research Society 68(10):11611176

De Armas J, Keenan P, Juan AA, McGarraghy S (2018) Solving large-scale time capacitated arc routing problems: from real-time heuristics to metaheuristics. Annals of Operations Research DOI 10.1007/s10479-018-2777-3

Dominguez O, Juan AA, Faulin J (2014) A biased-randomized algorithm for the two-dimensional vehicle routing problem with and without item rotations. International Transactions in Operational Research 21(3):375-398

Ferreira J, Pereira P, Filipe P, Afonso J (2011) Recommender system for drivers of electric vehicles. In: 2011 3rd International Conference on Electronics Computer Technology, vol 5, pp 244-248

Grasas A, Juan AA, Faulin J, de Armas J, Ramalhinho H (2017) Biased randomization of heuristics using skewed probability distributions: a survey and some applications. Computers \& Industrial Engineering 110:216-228 
GreenBiz (2019) One size doesn't fit all: How commercial EVs present unique challenges for charging infrastructure — GreenBiz. Available at: https://www.greenbiz.com/article/one-size-doesnt-fit-all-howcommercial-evs-present-unique-challenges-charging-infrastructure; accessed November 2019

Henderson D, Jacobson SH, Johnson AW (2003) The theory and practice of simulated annealing. In: Glover F, Kochenberger G (eds) Handbook of Metaheuristics, Springer US, Boston, MA, pp 287-320

Hill N, Brannigan C, Smokers R, Schroten A, Van Essen H, Skinner I (2011) The role of GHG emissions from infrastructure construction, vehicle manufacturing, and elvs in overall transport sector emissions. Tech. rep., European Commission Directorate-General Climate Action, available at: http://www.eutransportghg2050.eu/cms/assets/Uploads/Reports/EU-

Transport-GHG-2050-II-Task-2-FINAL-30Apr12.pdf, accessed: August 2018

Hof J, Schneider M, Goeke D (2017) Solving the battery swap station locationrouting problem with capacitated electric vehicles using an avns algorithm for vehicle-routing problems with intermediate stops. Transportation Research Part B: Methodological 97:102-112

Institute for Social-Ecological Research (2017) Transformations for sustainable development. [Available at: https://www.oeko.de/en/researchconsultancy/issues/sustainable-transport/e-mobility/; accessed August 2018

Jacobsen SK, Madsen OB (1980) A comparative study of heuristics for a twolevel routing-location problem. European Journal of Operational Research $5(6): 378-387$

Jouzdani J, Fathian M (2014) A linear mmtsp formulation of robust locationrouting problem: a dairy products supply chain case study. International Journal of Applied Decision Sciences 7(3):327-342

Juan AA, Faulin J, Grasman S, Riera D, Marull J, Mendez C (2011a) Using safety stocks and simulation to solve the vehicle routing problem with stochastic demands. Transportation Research Part C: Emerging Technologies 19(5):751-765, DOI 10.1016/j.trc.2010.09.007

Juan AA, Faulin J, Jorba J, Riera D, Masip D, Barrios B (2011b) On the use of monte carlo simulation, cache and splitting techniques to improve the clarke and wright savings heuristics. Journal of the Operational Research Society 62(6):1085-1097

Juan AA, Goentzel J, Bektaş T (2014) Routing fleets with multiple driving ranges: Is it possible to use greener fleet configurations? Applied Soft Computing 21:84-94

Juan AA, Pascual I, Guimarans D, Barrios B (2015) Combining biased randomization with iterated local search for solving the multidepot vehicle routing problem. International Transactions in Operational Research 22(4):647667

Juan AA, Mendez CA, Faulin J, de Armas J, Grasman SE (2016) Electric vehicles in logistics and transportation: A survey on emerging environmental, 
strategic, and operational challenges. Energies 9(2):86

Koç C, Bektaş T, Jabali O, Laporte G (2016) Thirty years of heterogeneous vehicle routing. European Journal of Operational Research 249(1):1 - 21

Lin C, Chow C, Chen A (2002) A location-routing-loading problem for bill delivery services. Computers \& Industrial Engineering 43(1):5-25

Mattila T, Antikainen R (2011) Backcasting sustainable freight transport systems for europe in 2050. Energy Policy 39(3):1241-1248

Mladenović N, Hansen P (1997) Variable neighborhood search. Computers \& Operations Research 24(11):1097-1100

Murty KG, Djang PA (1999) The us army national guard's mobile training simulators location and routing problem. Operations Research 47(2):175182

Nagy G, Salhi S (1996) Nested heuristic methods for the location-routeing problem. Journal of the Operational Research Society 47(9):1166-1174

Nagy G, Salhi S (2007) Location-routing: Issues, models and methods. European Journal of Operational Research 177(2):649-672

Pérez-Bernabeu E, Juan AA, Faulin J, Barrios BB (2015) Horizontal cooperation in road transportation: a case illustrating savings in distances and greenhouse gas emissions. International Transactions in Operational Research 22(3):585-606

Prins C, Prodhon C, Ruiz A, Soriano P, Calvo RW (2007) Solving the capacitated location-routing problem by a cooperative lagrangean relaxationgranular tabu search heuristic. Transportation Science 41(4):470-483

Quintero-Araujo CL, Gruler A, Juan AA, Faulin J (2017) Using horizontal cooperation concepts in integrated routing and facility-location decisions. International Transactions in Operational Research DOI 10.1111/itor.12479

Rath S, Gutjahr WJ (2014) A math-heuristic for the warehouse locationrouting problem in disaster relief. Computers \& Operations Research 42:2539

Salhi S, Rand GK (1989) The effect of ignoring routes when locating depots. European Journal of Operational Research 39(2):150-156

Sarıçiçek I, Akkuş Y (2015) Unmanned aerial vehicle hub-location and routing for monitoring geographic borders. Applied Mathematical Modelling 39(14):3939-3953

Schiffer M, Walther G (2017) The electric location routing problem with time windows and partial recharging. European Journal of Operational Research 260(3):995-1013

Schiffer M, Walther G (2018) Strategic planning of electric logistics fleet networks: A robust location-routing approach. Omega 80:31-42

Schiffer M, Schneider M, Laporte G (2018) Designing sustainable mid-haul logistics networks with intra-route multi-resource facilities. European Journal of Operational Research 265(2):517-532

Singh N, Shah J (2004) Managing tendupatta leaf logistics: an integrated approach. International Transactions in Operational Research 11(6):683699 
Tillman FA (1969) The multiple terminal delivery problem with probabilistic demands. Transportation Science 3(3):192-204

United States Environmental Protection Agency (2014) Sources of greenhouse gas emissions. Available at: https://www.epa.gov/ghgemissions/sourcesgreenhouse-gas-emissions, accessed: August 2018

Wager G, Whale J, Braunl T (2016) Driving electric vehicles at highway speeds: The effect of higher driving speeds on energy consumption and driving range for electric vehicles in australia. Renewable and Sustainable Energy Reviews 63:158 - 165

Wirasingha SG, Schofield N, Emadi A (2008) Plug-in hybrid electric vehicle developments in the us: Trends, barriers, and economic feasibility. In: 2008 IEEE Vehicle Power and Propulsion Conference, pp 1-8

Yang J, Sun H (2015) Battery swap station location-routing problem with capacitated electric vehicles. Computers \& Operations Research 55:217-232

Yıldız B, Arslan O, Karaşan OE (2016) A branch and price approach for routing and refueling station location model. European Journal of Operational Research 248(3):815-826

Yuksel T, Michalek JJ (2015) Effects of regional temperature on electric vehicle efficiency, range, and emissions in the united states. Environmental Science \& Technology 49(6):3974-3980, DOI 10.1021/es505621s 